\title{
3D nanoimprint for NIR Fabry-Pérot filter arrays: fabrication, characterization and comparison of different cavity designs
}

\author{
Duc Toan Nguyen ${ }^{1} \cdot$ Muath Ababtain $^{1,2} \cdot$ Imran Memon $^{1} \cdot$ \\ Anayat Ullah ${ }^{1,3} \cdot$ André Istock $^{1}$ - Carsten Woidt ${ }^{1} \cdot$ Weichang Xie $^{4}$. \\ Peter Lehmann ${ }^{4} \cdot$ Hartmut Hillmer ${ }^{1,5}$
}

Received: 18 December 2015/Accepted: 5 February 2016/Published online: 24 February 2016

(C) The Author(s) 2016. This article is published with open access at Springerlink.com

\begin{abstract}
We report on the fabrication of miniaturized NIR spectrometers based on arrays of multiple Fabry-Pérot (FP) filters. The various cavities of different height are fabricated via a single patterning step using high resolution 3D nanoimprint technology. Today, low-cost patterning of extended cavity heights for NIR filters using the conventional spin-coated nanoimprint methodology is not available because of insufficient coating layers and low mobility of the resist materials to fill extended cavity structures. Our investigation focuses on reducing the technological effort for fabrication of homogeneous extended cavities. We study alternative cavity designs, including a new resist and apply large-area 3D nanoimprint based on hybrid mold and UV Substrate Conformal Imprint Lithography (UV-SCIL) to overcome these limitations. We compare three different solutions, i.e. (1) applying multiple spin coating of the resist to obtain thicker initial resist layers, (2) introducing a
\end{abstract}

Duc Toan Nguyen

nguyen@ina.uni-kassel.de

1 Institute of Nanostructure Technology and Analytics, University of Kassel, Heinrich-Plett-Str. 40, 34132 Kassel, Germany

2 Present Address: King Abdulaziz City for Science and Technology (KACST), P.O. Box 6086, 11442 Riyadh, Kingdom of Saudi Arabia

3 Present Address: Faculty of Information and Communication Technology (ICT), Balochistan University of IT, Engineering and Management Sciences, Quetta 87300, Pakistan

4 Mesurement Technology, Department of Electrical Engineering and Computer Science, University of Kassel, Wilhelmshöher Alle 71, 34121 Kassel, Germany

5 CINSaT, Center for Interdisciplinary Nanostructure Science and Technology, University of Kassel, Heinrich-Plett-Str. 40, 34132 Kassel, Germany hybrid cavity (combination of a thin oxide layer and the organic cavity) to compensate the height differences, and (3) optimizing the imprint process with a novel resist material. The imprint results based on these methods demonstrate the implementation of NIR FP filters with high transmission intensity (best single filter transmission $>90 \%)$ and small line widths $(<5 \mathrm{~nm}$ in full width at half maximum).

Keywords Near-infrared $\cdot$ Spectrometer $\cdot 3 \mathrm{D}$ nanoimprint · Nanospectrometer · Fabry-Pérot filter . Miniaturization

\section{Introduction}

Optical spectroscopy referring to the study of absorption and emission spectra of matter in the visible, near infrared (NIR) and ultraviolet (UV) spectral range, is a valuable measurement method. It has been extensively studied and widely applied in many fields such as in medicine or in agriculture (Berger et al. 1997; Schrader et al. 1999; Clevers 1999). For many applications, low fabrication cost, high resolution and capability for strong miniaturization of the optical spectrometer, are crucial demands, especially for networked sensing systems. A strongly miniaturized spectrometer based on FP filter arrays and sophisticated deposition processes has been presented (Correia et al. 2000; Wolffenbuttel 2005). We use a similar concept, but replace the multiple deposition steps to generate various FP cavity heights by a single step: 3D nanoimprint. This enables the various cavity heights to differ from each other in the nm region. Since 3D nanoimprint is applied we call the device optical nanospectrometer (Wang et al. 2013; Albrecht et al. 2012). It consists of a static FP filter array 
and a corresponding detector array. All FP filters are using two identical dielectric Distributed Bragg Reflectors (DBRs) designed for a specific wavelength range. However, each filter reveals a different resonance cavity height (defined by the nanoimprinted resist) in between the DBRs and defines a different small spectral transmission band (called filter transmission line below). Up to now, our research was focused on the visible spectral range between 460 and $700 \mathrm{~nm}$ involving corresponding cavity heights between 150 and $270 \mathrm{~nm}$ (Albrecht et al. 2012).

Each DBR consists of a periodic sequence of thin dielectric films with alternating high and low refractive indices. The optical thickness of each thin film in the DBR is a quarter of the design wavelength in the respective material. The DBR reveals a spectral range of high reflectivity (the so-called stopband) and these two DBRs form a resonator and the space in between is called FP cavity. The thickness of each cavity is directly proportional to the corresponding spectral filter line for a considered cavity material for each individual filter. The UV Substrate Conformal Imprint Lithography (UV-SCIL) methodology uses hybrid molds, enables to generate 2D structures and was developed by Philips Research and Süss MicroTec AG (Ji et al. 2010). We were enlarging the SCIL methodology to $3 \mathrm{D}$ and very high resolution in the vertical direction. Thus, we are now able to apply 3D SCIL to fabricate different cavity heights in a single step with high vertical resolution (Albrecht et al. 2012).

To address the NIR spectral range with our nanospectrometer, it is required to generate cavity heights up to $1 \mu \mathrm{m}$ thickness. One of the main challenges is to produce the homogeneous extended cavities using 3D nanoimprint with high precise vertical resolution. The height resolution of the cavity structures depends sensitively on the quality of the initially spin-coated UV-curable resist layers and the available viscosity of the UV-curable resist (Vogler et al. 2007; Atobe et al. 2010). Considering a distinct viscosity the applied spinning frequency is limiting the resist layer thickness. High thicknesses require low spin frequencies; however, low frequencies will strongly degrade the homogeneity of the resist layer and the quality of the imprinted structures.

In this paper, we compare three methods for structuring the extended cavities to overcome the challenges mentioned above: (1) using multiple spin coating of the resist to obtain a thicker initial resist layer, (2) using a "hybrid" cavity combining oxide layers and the UV-curable resist to accumulate the required cavity heights and (3) optimizing the nanoimprint process with a novel resist material.

The three methods are applied to fabricate FP filter arrays for the above mentioned spectral range between 1.4 and $1.5 \mu \mathrm{m}$ and the experimental results are finally compared in detail, respectively.

\section{Experimental work}

For the feasibility of our nanospectrometer, a high transmission intensity and a small line width (FWHM) of the filter transmission line are required. These optical parameters strongly depend on the quality of the cavities, i.e. the thickness and homogeneity of cavities. Therefore, the cavity thicknesses and roughness are investigated to compare the different structural designs. In this chapter, we report our technological effort in structuring homogeneous extended cavities and characterization process of FP filters in NIR spectral range.

\section{Structuring homogeneous extended cavities}

To overcome a poor resist layer quality after applying the spin coating process and to obtain homogeneous extended cavities for fabrication of FP filter arrays in NIR spectral range, our three different methods are displayed in Fig. 1.

1. Multi-spin coating method The multiple layer cavities are structured using subsequently several spin coating steps. The thickness of each resist layer in each individual spin coating process is $d_{1}(\mathrm{~nm}), d_{2}$ $(\mathrm{nm}), \ldots d_{n}(\mathrm{~nm})$. Thus, the total cavity thickness is described $d=d_{1}+d_{2}+\cdots d_{n}$ ideally. Each layer is cured by UV light for a few minutes except the last layer. The spin coating frequency depends on the desired individual thickness. The last resist layer has to meet the required thickness for the SCIL process to receive the desired cavity structure.

2. Hybrid cavity method Thin oxide layers having a similar refractive index as the UV-curable resist are used to enlarge the total cavity height. Two thin oxide layers are embedding the resist layer which is imprinted. In this case, the three layers comprise the cavity.

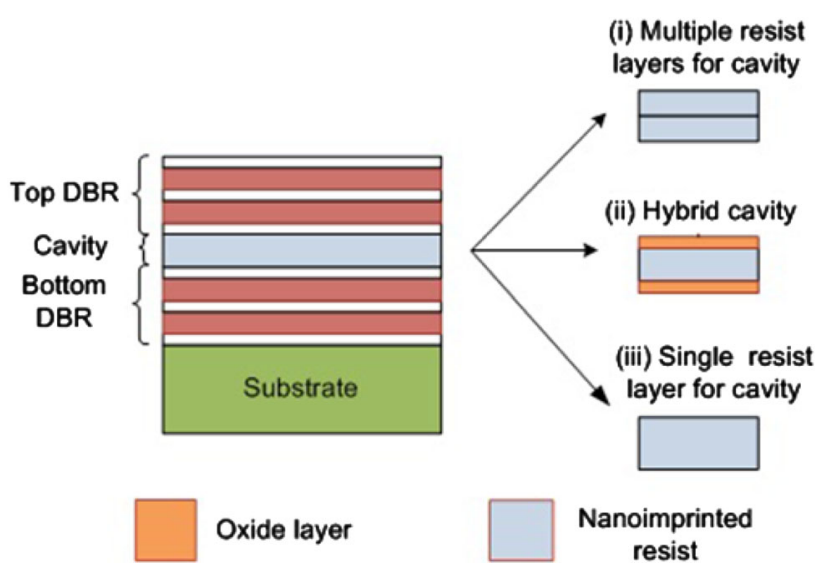

Fig. 1 Schematic of structuring FP filter cavities using three different methods 
3. Novel resist material The imprint process is modified and improved by applying a newly developed UV-NIL resist for the use of PDMS based stamps with freely adjustable film thickness (mr-NIL210), the full desired thickness of the initial resist layer can be achieved in a single spin coating step at high spin frequencies guaranteeing a smooth and homogeneous surface layer.

\section{Fabrication and characterization process of FP filter arrays}

For the third method, the fabrication process of FP filter arrays comprises the following steps: first, the bottom DBR is deposited on a detector array or on a transparent substrate which is combined with a detector array afterwards. Next, the 3D nanoimprint process is performed using a 3D nanoimprint template that contains negative cavity structures. Finally, the top DBR is deposited on the top of the nanoimprinted cavities. This process is applied to fabricate FP filter arrays using the novel resist. In case of using multiple spin coating or hybrid cavity method, some additional steps have to be included to process the extended cavity structures: Multi-spin coating steps to define the multiple layer cavity structures as well as the depositions of thin oxide layers, respectively. In this paper, we used spin coating twice, thus, a double resist layer was generated.

The FP filter arrays are designed for the 1.4-1.5 $\mu \mathrm{m}$ spectral range applying the three different designs to obtain extended cavities. 9.5 periods $\mathrm{Si}_{3} \mathrm{~N}_{4} / \mathrm{SiO}_{2}$ is deposited by Plasma Enhanced Chemical Vapor Deposition (PECVD) for the bottom and top DBR. These DBRs are deposited at a temperature of $120{ }^{\circ} \mathrm{C}$ to avoid the effect of higher temperature to the resist. Two different UV-curable resists are used. The resist mr-UVCur06 is used for the hybrid cavity method and for the method involving multiple spin coatings. The novel resist mr-NIL210 is used for the single spin coating process. Both products are commercially available from micro resist technology $\mathrm{GmbH}$, Germany. The thin oxide layers used for the hybrid cavity consist of $\mathrm{SiO}_{2}$ since it has a comparable refractive index to the resists mr-UVCur06. Figure 2 depicts the spectral variation of the refractive index for $\mathrm{Si}_{3} \mathrm{~N}_{4}, \mathrm{SiO}_{2}, \mathrm{mr}-\mathrm{NIL} 210$ and mrUVCur06. In the spectral range of interest, the difference in refractive index between $\mathrm{SiO}_{2}$ and mr-UVCur06 is smaller than 0.1 .

The optical characterization of the FP filter arrays is carried out with a free beam broad band confocal setup which comprises an illumination system, a collimation and magnification system, a sample stage and a data recording and analysis system (Mai et al. 2012; Mai 2012).

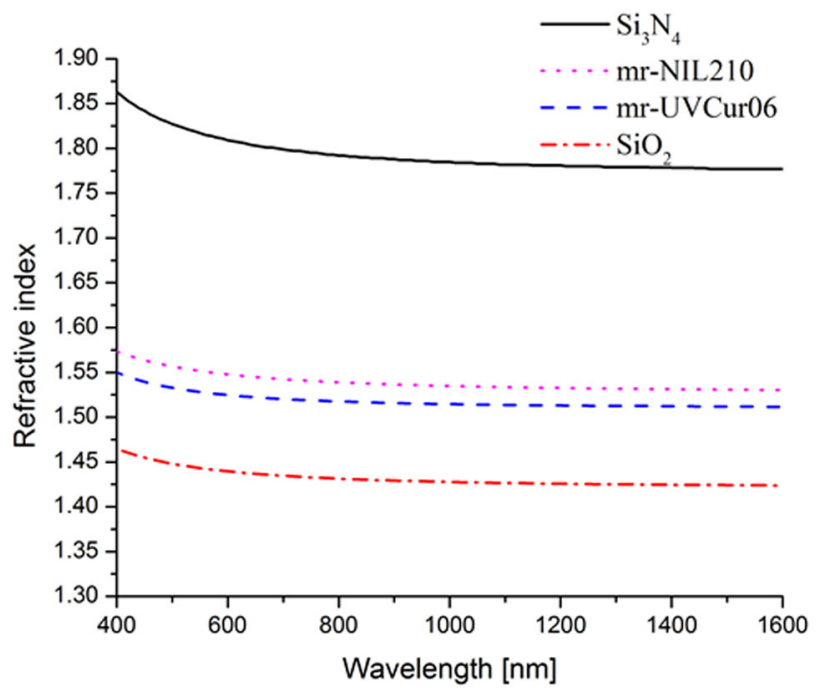

Fig. 2 Schematic of the refractive indices of $\mathrm{SiO}_{2}, \mathrm{Si}_{3} \mathrm{~N}_{4}, \mathrm{mr}-$ UVCur06 and mr-NIL210

For the thermal properties investigation, the shrinkage of the imprinted structures is characterized by white-light interferometry (WLI) Zygo NewView 5000 system from Zygo Corporation (USA) with maximum vertical step height of $100 \mu \mathrm{m}$ and vertical resolution of $0.1 \mathrm{~nm}$. A thin layer of platinum is coated on the top of imprinted structure to get high reflectivity under WLI.

The surface roughness of the resist layer is measured by atomic force microscopy (AFM) Dualscope DS95 system from DME Nanotechnologie GmbH, Germany using tapping mode.

\section{Topography measurement of nanoimprinted cavities}

The nanoimprinted cavities are characterized by two different optical measuring instruments. The first one is a commercial confocal microscope (NanoFocus $\mu$ surf $320 \times$ S) with a $50 \times$ magnification and a numerical aperture of 0.95 . Confocal microscopes utilize pinhole apertures to achieve axial depth discrimination eliminating of out-of-focus light. They can reach high axial resolution up to the nanometer range. Our instrument provides a height resolution of $2 \mathrm{~nm}$ and a field of view of $320 \times 320 \mu \mathrm{m}$, which results in a lateral resolution of $0.33 \mu \mathrm{m}$ at a pixel number of $984 \times 984$. The depth response recorded by a CCD during a depth scan is analyzed to determine the topography data. Figure $3 \mathrm{a}$, b shows a measured three-dimensional topography of the nanoimprinted cavities and one corresponding cross section. Due to a limited measurement field, only a $6 \times 6$ filter array out of a $8 \times 8$ filter array could be measured. Each filter has lateral dimensions of $40 \times 40 \mu \mathrm{m}$, the vertical dimension of the imprinted structures vary in between 365 and 
$530 \mathrm{~nm}$. In Fig. 3a different heights of cavities can be recognized. The cross section represented in Fig. $3 \mathrm{~b}$ allows to determine the step heights.

Figure $4 \mathrm{a}, \mathrm{b}$ displays results obtained with the second measuring instrument: a self-designed and self-built Linnik type white-light interferometer (Lehmann et al. 2014).
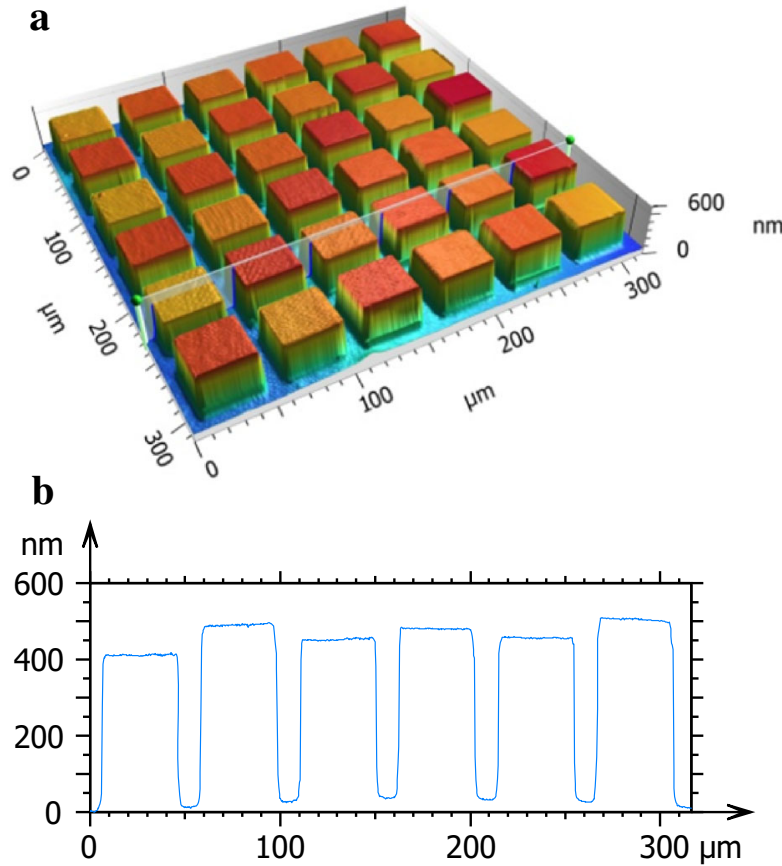

Fig. 3 a Measurement results of an array including a single mrNIL210 cavity layer obtained by a confocal microscope. b Height profile of the cross section indicated in $\mathbf{a}$

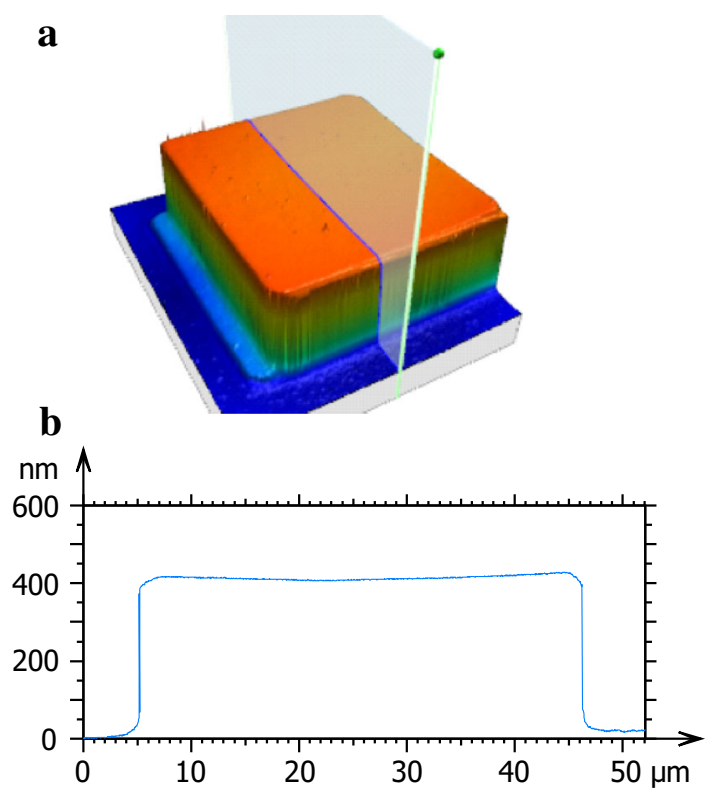

Fig. 4 a Measurement results obtained by a Linnik type white-light interferometer providing a high lateral resolution. $\mathbf{b}$ Height profile of the cross section indicated in Fig. 4a
White-light interferometers use optical coherence phenomena and interference to generate phase and amplitude modulated signals from which the surface topography can be obtained. The interference correlogram of each camera pixel shows a height dependent axial shift according to the corresponding location on the measurement object. The signals can be evaluated by determination of the peak position of the coherence envelope. Analyzing the phase information enhances the axial resolution to the subnanometer range. The white-light interferometer used has a magnification of $100 \times$, a numerical aperture of 0.9 and a field of view of $70 \times 52 \mu \mathrm{m}$. Due to the limited depth of focus of the objective lenses of $1.5 \mu \mathrm{m}$ color LEDs can be used for illumination. For a blue LED illumination implemented in our setup a lateral resolution of $0.31 \mu \mathrm{m}$ results. The measurement results, obtained from the same cavity mesa (the third one in Fig. 3b) are represented in Fig. 4b.

The measured step height in Fig. 4b agrees quite well with the result of the confocal microscope except for a few nanometers. For the top layer an rms roughness value of $0.57 \mathrm{~nm}$ is obtained from the interferometer result.

\section{Results and discussion}

\section{Optical characterization of FP filter arrays}

Figures 5, 6, 7 show optical transmission spectra of FP filter arrays designed and fabricated by the three different cavity structuring methods, respectively. In these figures, we show a selection of some typical filter transmission lines (representatives) out of 64 spectrally different lines to

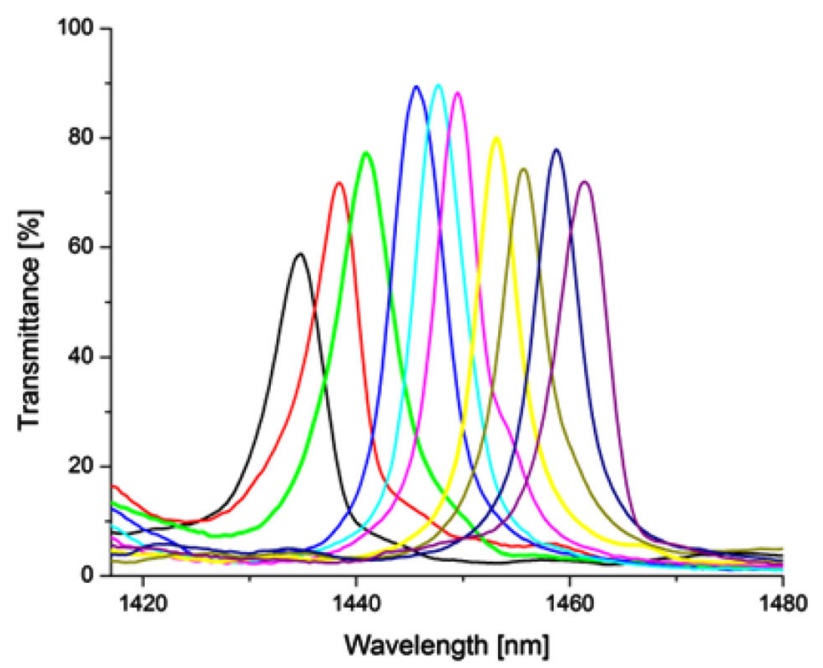

Fig. 5 Optical transmission spectra of FP filter arrays using spin coating twice for the cavity layer 


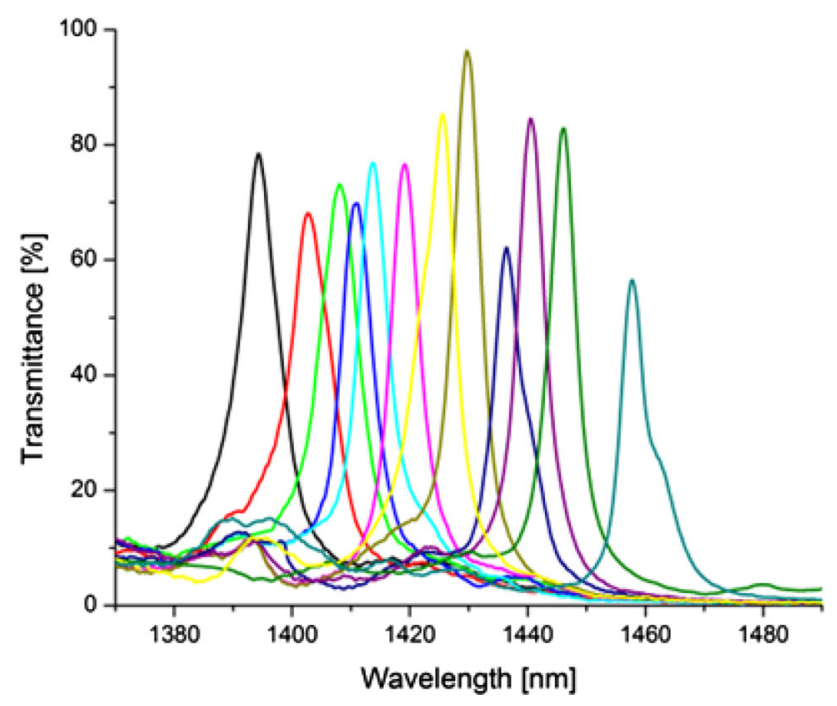

Fig. 6 Optical transmission spectra of FP filter arrays including hybrid cavities

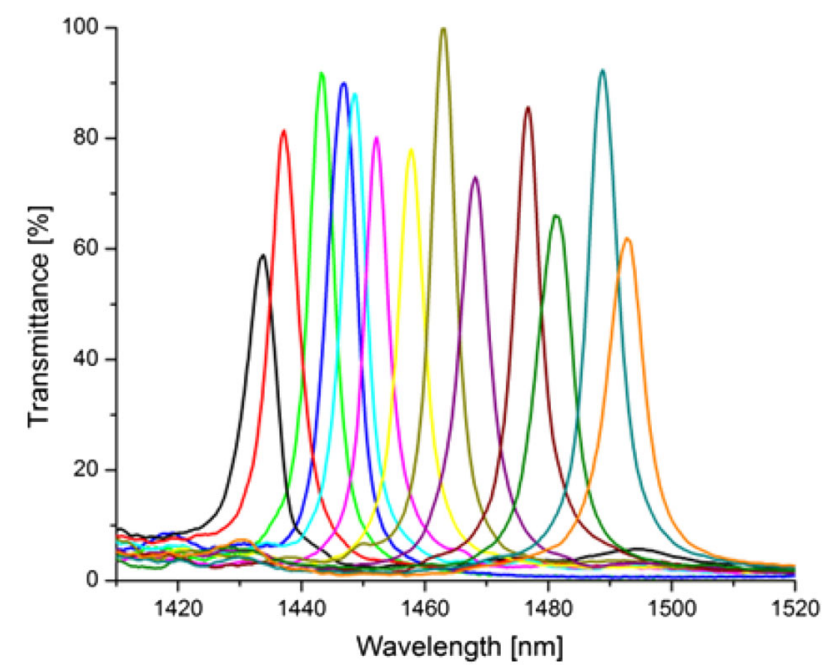

Fig. 7 Optical transmission spectra of FP filter arrays using the resist mr-NIL210 for the cavity layer

avoid overloading. High maximum transmissions of all filter transmission lines up to $>90 \%$ are observed and the average transmission value is well above $70 \%$. The FWHM of all filter transmission lines is between 4.7 and $8.2 \mathrm{~nm}$ and the smallest FWHM is achieved at $1450 \mathrm{~nm}$ using the novel resist.

This provides a resolution, which is defined as the ratio of the transmission wavelength and the FWHM, of $>300$.

\section{Comparison of optical properties of three FP filter fabrication methods}

In the following we are comparing the three methods with respect to optical properties. In Figs. 8, 9, 10 the results for

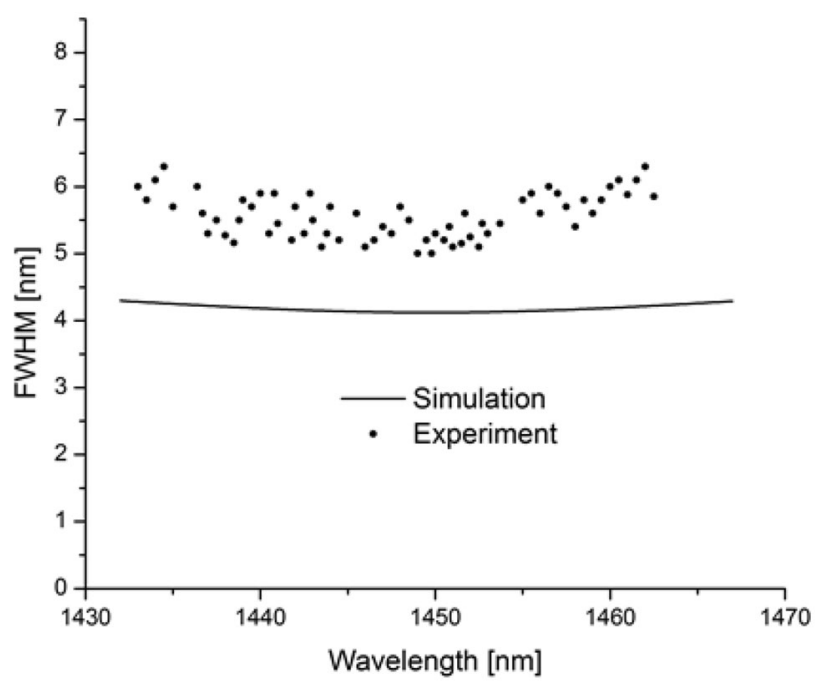

Fig. 8 Comparison of measured and simulated FWHM of FP filter arrays using spin coating twice for the cavity layer

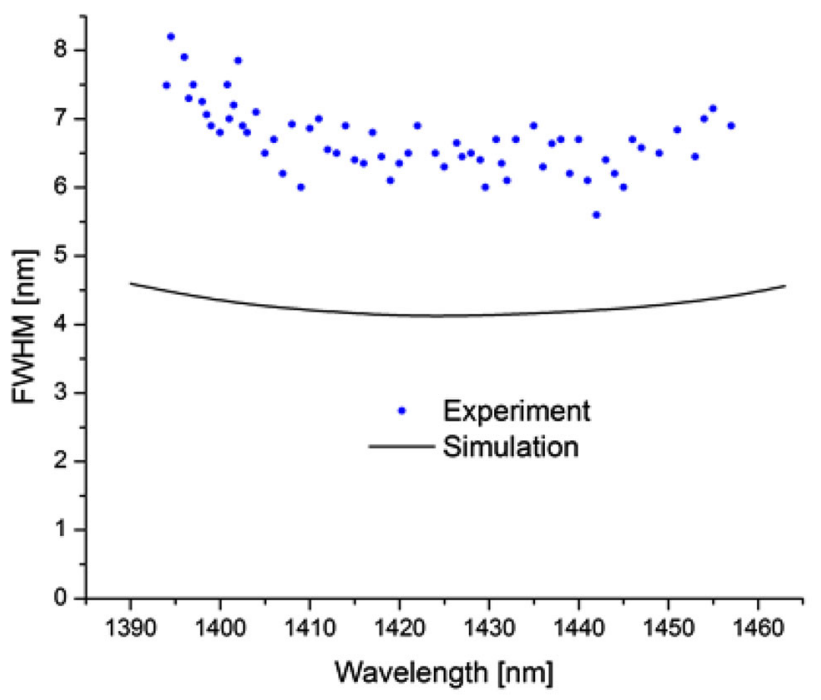

Fig. 9 Comparison of measured and simulated FWHM of FP filter arrays including a hybrid cavity

the FWHM obtained by theoretical model calculation and experimental characterization for each method are summarized and compared. For the simulation we used a tool based on a Transfer Matrix Method and the material parameters with the spectral variation of the refractive index and extinction coefficient were obtained experimentally by measuring the respective pure material samples on Si wafers by spectroscopic ellipsometry.

Figures 8, 9, 10 show the spectral variations of the experimental and simulated FWHM. The observed behavior in the absolute values is similar comparing the three applied methodologies. For the simulation, the minimum FWHM is located in the center of the stopband, 
larger values are obtained for the filter transmission lines located at the borders of the stopband. This variation we can also observe in experimental results with a small deviation. The reason for this FWHM variation is due to the fact that the reflectivity of the DBR mirrors is largest in the center of the stopband. The more the filter lines are shifted towards the borders of the stopband, the lower the reflectivity and, thus, the larger the FWHM of the lines.

Hence, the experimental and theoretical results are in agreement with our studies on InP multiple air-gap FP filters (Irmer et al. 2003; Römer 2005).

The Figs. 8, 9, 10 show that the experimental FWHM data are generally larger than the simulated results. This phenomenon is partly due to the fact that in simulation we neglected (1) influence of non horizontal wave fronts in the cavities during the measurements and (2) the interface roughnesses which exist in the heterostructure and might sum up with the number of cavity layers. In addition we neglected (3) absorption of resist material and (4) Rayleigh scattering.

The surface roughness of layered heterostructures is investigated on the resist mr-UVCur06 using AFM. We observed an rms of below $4 \mathrm{~nm}$ for a single layer and $8 \mathrm{~nm}$ for a double layer. This would be in qualitative agreement with our observations, since in layered heterostructures,

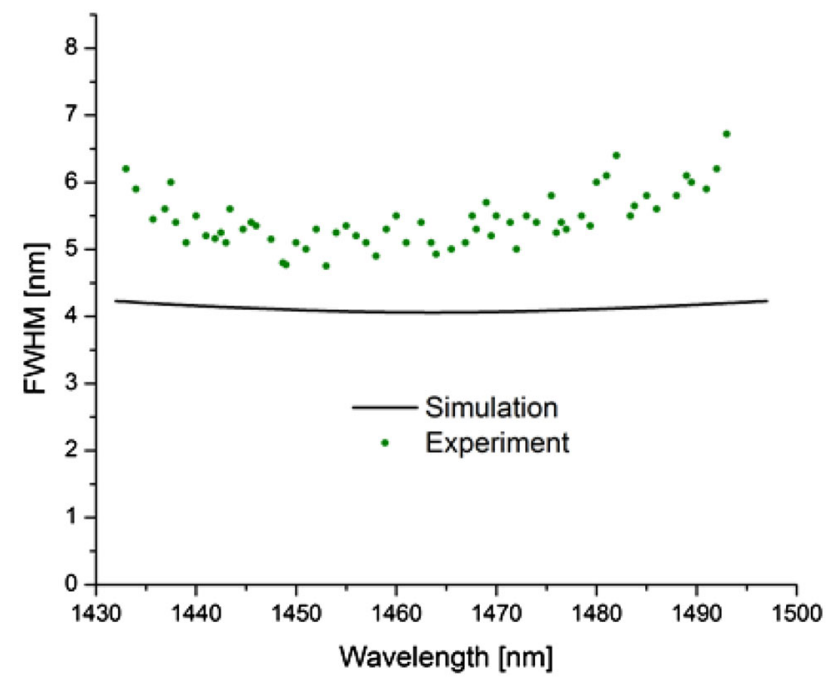

Fig. 10 Comparison of measured and simulated FWHM of FP filter arrays using the resist mr-NIL210 for the cavity layer these measured surface roughnesses are incorporated and slightly modified into interface roughnesses. Furthermore, surface, respectively, interface roughness also exists for $\mathrm{SiO}_{2}$ layers deposited by PECVD. Previously, we reported on our measurements on $\mathrm{SiO}_{2}$ layers deposited by different technological methods recorded by a profilometer and AFM revealing an rms of below $3 \mathrm{~nm}$ (Amirzada et al. 2015). This result corresponds to our situation. Thus, the larger the number of layers included in our cavity, the more interfaces are existing and the higher the finally accumulating interface roughness.

In addition to these roughnesses on a short length scale (AFM) we observed height variations on a longer length scale in the lateral directions using white-light interferometry, e.g. Figure 4 shows the height profile in lateral resolution of a cavity surface using the resist mr-NIL210. A variation of several $\mathrm{nm}$ is observed for this height profile. Since we measured the transmission of the whole cavity area in our optical characterization the variation is affecting the optical properties of FP filters. Therefore, larger FWHM and lower transmitted intensity of the experimental results are obtained compared to the simulation as discussed above.

Table 1 reveals the average value of the simulated and experimental FWHM of the filter transmission lines of each method, respectively. These calculations are based on all available data (64, for each method). The FWHM is largest for the method using a hybrid cavity in both simulated and experimental results. The reason behind this phenomenon is that the small mismatch of refractive index of $\mathrm{SiO}_{2}$ and mr-UVCur06 in the cavity structure affects the interference inside the cavity, i.e. causing larger FWHM. In addition, the number of interfaces in the cavity structure affects also the FWHM as we see in the multiple spin coating method which has the FWHM larger than the novel resist method and smaller than the hybrid cavity method for experimental as well as simulation results. The larger the number of involved interfaces in the cavity, the larger the FWHM.

In Figs. 11, 12, 13, we compare the simulated and the experimental transmittance at the maximum of the filter transmission line for each method. The experimental results are in most cases smaller than the simulated values. This is due to the limitations in theoretical model as we mentioned above. Furthermore, we measured zero for the

Table 1 Average FWHM obtained for each FP filter array fabrication method

\begin{tabular}{llll}
\hline $\begin{array}{l}\text { Specific characteristics of the } \\
\text { technological method used }\end{array}$ & $\begin{array}{l}\text { Measured FWHM (nm) of the } \\
\text { filter transmission line }\end{array}$ & $\begin{array}{l}\text { Simulated FWHM (nm) of the } \\
\text { filter transmission line }\end{array}$ & $\begin{array}{l}\text { Number of interfaces } \\
\text { involved in the cavity }\end{array}$ \\
\hline Hybrid cavity & $6.6 \pm 0.5$ & 4.3 & 4 \\
Multiple spin coating & $5.6 \pm 0.3$ & 4.2 & 3 \\
Novel resist & $5.4 \pm 0.4$ & 4.1 & 2 \\
\hline
\end{tabular}




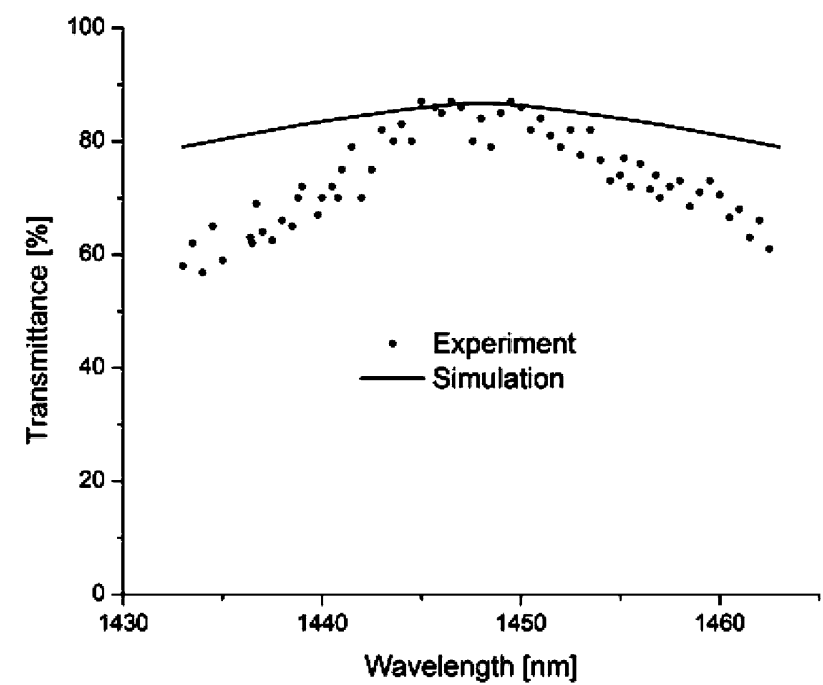

Fig. 11 Comparison of simulated and experimental transmittance of the FP filter lines. The filters have been fabricated using multiple spin coating for the cavity layer

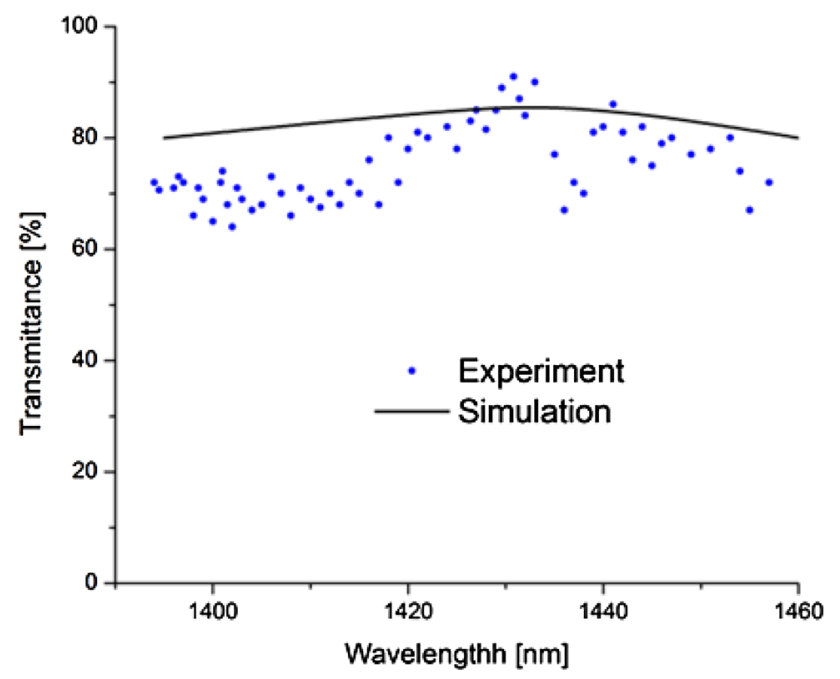

Fig. 12 Comparison of simulated and experimental transmittance of the FP filter lines. The filters have been fabricated using the hybrid cavity method

optical absorption in the considered spectral range for the involved resist materials using spectroscopic ellipsometry. Thus, our theoretical model calculations have been carried out without absorption within the resist materials. However, absorption can never be completely zero in reality. This is an additional reason for the difference in the absolute values. Figures 11, 12, 13 reveal that the simulated transmittance of three methods is between 79 and $91 \%$. The lowest transmitted intensity is obtained for the hybrid cavity design since the presence of $\mathrm{SiO}_{2}$ in the cavity layer affects the interference inside the cavity as discussed above. This $\mathrm{SiO}_{2}$ layer also absorbs light and,

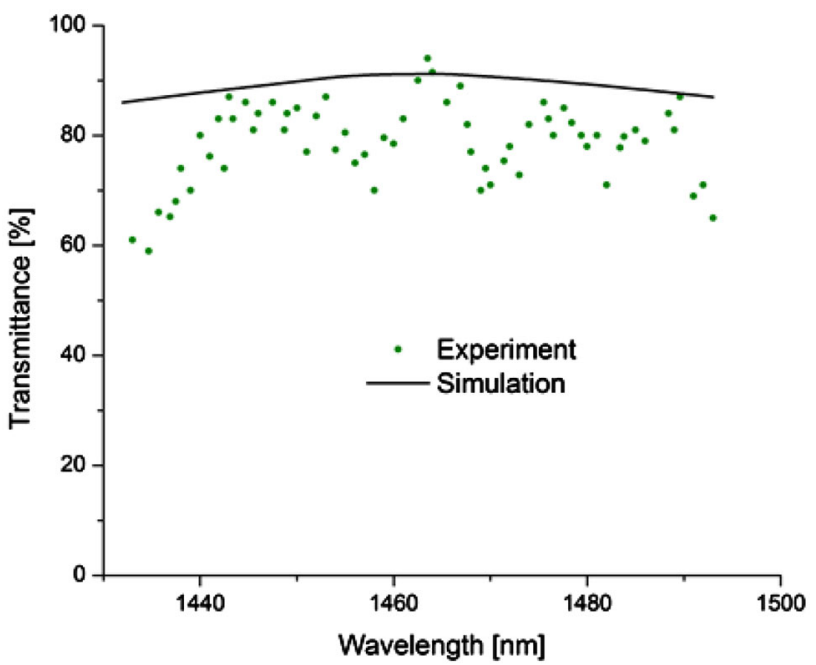

Fig. 13 Comparison of simulated and experimental transmittance of the FP filter lines. The filters have been fabricated using the resist mrNIL210

therefore, reduces the transmitted intensity of the filter transmission lines. The experimental transmittance measured for samples fabricated by three methods ranges between 55 and $93 \%$. Fluctuation in the experimental data and mismatch of the shapes in simulated and experimental transmittance profiles are mainly observed for the hybrid cavity and for the cavity including the novel resist. This is due to the reasons mentioned before. The cavity material mr-NIL210 might have wavelength dependent absorption which has been neglected in the simulation, this absorption could lead to the undulation presented in the experimental result in Fig. 13. In addition the surface roughness of the cavities could lead to wavelength dependent scattering. Moreover, the height variation of the imprinted cavities measured on a larger length scale, as discussed at the beginning of this paragraph, affects the transmitted intensity of the filters.

For each of the three different cavity designs we fabricated several filter arrays. For this publication we selected representatives for each cavity design (Figs. 5, 6, 7, 8, 9, $10,11,12,13)$. The selected arrays include most details and are representative for the other arrays for each cavity design.

\section{Comparison of the technological effort for the three FP filter fabrication methods}

By applying the multiple coating and the hybrid cavity design, we could avoid operating the conventional mrUVCur06 resist at unfavorable spin frequencies. Therefore, we could reduce the degradation in homogeneity of the resist layer which enables a better 3D nanoimprint result 
(the option using single spin coating of conventional mrUVCur06 provided incompletely filled structures and is not included in this paper). However, these two methods take time and extra cost due to multiple coating steps of the resist layer or multiple depositions of thin oxide layers. Moreover, these two methods take more effort to control the total cavity thickness since each individual layer generates an error which accumulate to an error of the total cavity thickness and lead to a shift of the filter transmission line at the end. The consequence is observed in the optical transmittance property of FP filters including the hybrid cavity shown in Fig. 9. The optical transmission spectra of the filter arrays are shifted to smaller wavelength since the total cavity thickness is lower than the designed thickness. In contrast, the third method using the novel material mrNIL210 saves time and cost in fabrication of FP filter arrays since it requires only a single spin coating step to define the desired initial resist layer thickness and also reduces the effort in controlling the cavity thicknesses. The higher the number of potential error sources the higher the required effort to control the thickness of the total cavities in practice.

\section{Discussion about thermal properties and mechanical properties of different FP filter designs}

The optical properties of FP filters are not only depending on the interface roughness of the layers included in the cavities, but they are also affected by the process conditions during the top DBR fabrication. This process affects the imprinted cavity by (1) temperature, (2) plasma and (3) pressure conditions. All three parameters influence the shrinkage of the resist layer and, therefore, might affect the spectral position of the filter lines at the end. The imprinted structures are measured before and after the top DBR deposition. We observed a shrinkage of $13.7 \%$ for structures defined by the double spin coating method using mrUVCur06, $7.9 \%$ for applying the novel material mrNIL210 and $4.3 \%$ for structures defined by the hybrid cavity method combining mr-UVCur06 and $\mathrm{SiO}_{2}$. The reasons behind the lower shrinkage applying the hybrid cavity method are on one hand the partial shrinkage of the imprinted structures already occurring during the deposition of the top $\mathrm{SiO}_{2}$ layer for the hybrid cavity and on the other hand the smaller volume of resist existing in the cavity structures. The latter is caused by the fact that the $\mathrm{SiO}_{2}$ layer is counted to the cavity thickness, but only the polymer is shrinking. To end up with the targeted cavity heights, the shrinkages have to be considered during the design process of the structures.

Next the sensitivity of the spectrometer including FP filter arrays against temperature changes is considered. Assuming similar thermal conductivities of the involved material layers, the interfaces included in the heterostructure might reduce the overall thermal conductivity across the interfaces considerably. The literature (Abramson and Tien 2002) does not yet reveal a clear and distinct conclusion as well as a full understanding of the impact of interfaces. However, most of the studies presented up to now in the literature reveal a rule of thumb: the thermal conductivity is decreasing with rising number of interfaces (Abramson and Tien 2002) and the first interface most probably is providing the largest heat resistance compared to the last interface of multiple interfaces. Therefore, having only few interfaces, the difference in thermal resistivity between a single interface and three interfaces is expected to be noticeable. Thus, with increasing number of interfaces we might have a rising non-optimum heat transfer to the heat sink. This again could give another reason for a preference of the method using the novel resist and less involved interfaces.

In addition, the multiple layer designs could give the chance for a reduction of the overall temperature expansion coefficient, in case materials of positive and negative coefficients are used in combination. However, it is hard to find materials of negative thermal expansion coefficients like Yttrium oxide. Thus, the probability to benefit from this advantage of multiple material cavity designs is extremely low, which again supports the preference of the method involving the novel resist.

The above mentioned statements also hold as a preliminary characterization for trends in mechanical stability. In addition, the mechanical stability is expected to be strongly influenced by the number of interfaces and material

Table 2 Brief compilation of the criteria in each cavity structuring method

\begin{tabular}{llc}
\hline $\begin{array}{l}\text { Criteria } \\
\text { Method }\end{array}$ & Technological effort & Properties \\
\hline $\begin{array}{c}\text { Multiple } \\
\text { spin } \\
\text { coating }\end{array}$ & $\begin{array}{c}\text { More difficult to control the total cavity thickness due to the } \\
\text { multiple spin coating steps; process more expensive }\end{array}$ & $\begin{array}{c}\text { Better optical properties than hybrid cavity method and weaker } \\
\text { optical properties compared to novel resist method }\end{array}$ \\
$\begin{array}{c}\text { Hybrid } \\
\text { cavity }\end{array}$ & $\begin{array}{c}\text { More difficult to control the total cavity thickness due to multiple } \\
\text { depositions; process more expensive }\end{array}$ & $\begin{array}{c}\text { Weakest optical properties compared to methods using } \\
\text { multiple spin coating and novel resist for the cavity }\end{array}$ \\
$\begin{array}{c}\text { Novel resist } \\
\text { Saves time during the process; easier to control the resist } \\
\text { thickness. Cheapest process of the three methods }\end{array}$ & $\begin{array}{c}\text { Best optical properties of the three methods } \\
\end{array}$
\end{tabular}


adhesion of two materials forming a heterointerface. If heterostructures including soft and hard matter in combination like in all our structures we have a complex situation regarding mechanical stability in the point of view of a classical stress-rupture test. In a preliminary estimation there is not yet a clear preference for one of our structure designs.

Summarizing the result and discussion up to this point, the method involving the novel resist is superior to the other two methods for nearly all aspects including optical properties and thermal properties. The criteria of each method are summarized in Table 2 .

\section{Conclusion}

Different methods for structuring homogeneous extended cavities have been implemented, namely the multiple spin coating method, the hybrid cavity method and the novel resist method. Those methods were applied to fabricate FP filter arrays in the spectral range between 1.4 and $1.5 \mu \mathrm{m}$. Surface topography and optical properties have been measured. Finally, optical, thermal and mechanical behavior was discussed and compared for each of the three methods.

A high maximum filter line transmission up to $>90 \%$ and average transmission values in the spectral filter line maximum above $70 \%$ have been observed. The smallest FWHM of $4.7 \mathrm{~nm}$ has been observed for structures involving the novel resist. Theoretical model calculations have been used to calculate transmission spectra revealing a maximum transmittance and FWHM as a function of wavelength. Both reveal a reasonable agreement with respective experimental data obtained for all three methods. This qualitative agreement strongly supports the validity and consistency of technological fabrication, characterization and simulation. The optical and thermal properties and the roughness data are found to be best for the fabrication method involving the novel resist $\mathrm{mr}-$ NIL210 deposited as a single layer.

FP filter arrays for the spectral range between 1.4 and $1.5 \mu \mathrm{m}$ have been implemented for the first time demonstrating that it is feasible to fabricate homogeneous extended cavities with 3D nanoimprint technology. Thus, low cost, strongly miniaturized, high optical resolution FP filter arrays for the NIR spectral range as important elements of nanospectrometers are achievable.

Acknowledgments The authors would like to thank to D. Gutermuth, J. Krumpholz, A. Dück for technical support and Y. Shen, R. Kolli and T. Meinl for stimulating scientific discussion. The authors also would like to thank Dr. M. Thesen from micro resist technology $\mathrm{GmbH}$ for technical support and discussion. The financial support of the Ministry of Education and Training (Vietnam) is gratefully acknowledged.
Open Access This article is distributed under the terms of the Creative Commons Attribution 4.0 International License (http:// creativecommons.org/licenses/by/4.0/), which permits unrestricted use, distribution, and reproduction in any medium, provided you give appropriate credit to the original author(s) and the source, provide a link to the Creative Commons license, and indicate if changes were made.

\section{References}

Abramson AR, Tien C (2002) Interface and strain effects on the thermal conductivity of heterostructures: a molecular dynamics study. J Heat Transf 124:963-970. doi:10.1115/1.1495516

Albrecht A, Wang X, Mai HH et al (2012) High vertical resolution 3D nanolmprint technology and its application in optical nanosensors. Nonlinear Opt Quantum Opt 43:339-353

Amirzada MR, Tatzel A, Viereck V, Hillmer H(2015) Surface roughness analysis of $\mathrm{SiO} 2$ for PECVD, PVD and IBD on different substrates. Appl Nanosci. doi:10.1007/s13204-015-0432-8

Atobe H, Hiroshima H, Wang Q (2010) Evaluation of viscosity characteristics of spin-coated UV nanoimprint resin. Jpn J Appl Phys. doi:10.1143/JJAP.49.06GL10

Berger AJ, Itzkan I, Feld MS (1997) Feasibility of measuring blood glucose concentration by near-infrared Raman spectroscopy. Spectrochim Acta Part A Mol Biomol Spectrosc 53:287-292

Clevers JGPW (1999) The use of imaging spectrometry for agricultural applications. ISPRS J Photogr Remote Sens 54:299-304. doi:10.1016/S0924-2716(99)00033-7

Correia JH, de Graaf G, Kong SH et al (2000) Single-chip CMOS optical microspectrometer. Sens Actuators A Phys 82:191-197. doi:10.1016/S0924-4247(99)00369-6

Irmer S, Daleiden J, Rangelov V et al (2003) Ultralow biased widely continuously tunable Fabry-Pérot filter. IEEE Photonics Technol Lett 15:434-436

Ji R, Hornung M, Verschuuren MA et al (2010) UV enhanced substrate conformal imprint lithography (UV-SCIL) technique for photonic crystals patterning in LED manufacturing. Microelectron Eng 87:963-967. doi:10.1016/j.mee.2009.11.134

Lehmann P, Niehues J, Tereschenko S (2014) 3-D optical interference microscopy at the lateral resolution. Int J Optomech 8:231-241. doi:10.1080/15599612.2014.942924

Mai HH (2012) Characterization of Novel Fabry Pérot Filter Arrays for Nanospectrometers in Medical Applications. PhD thesis, University of Kassel

Mai HH, Albrecht A, Woidt C et al (2012) 3D nanoimprinted FabryPérot filter arrays and methodologies for optical characterization. Appl Phys B 107:755-764. doi:10.1007/s00340-012-5063-0

Römer F (2005) Charakterisierung und Simulation optischer Eigenschaften von mikromechanisch abstimmbaren Filterbauelementen. $\mathrm{PhD}$ thesis, University of Kassel

Schrader B, Dippel B, Erb I et al (1999) NIR Raman spectroscopy in medicine and biology: results and aspects. J Mol Struct 480-481:21-32. doi:10.1016/S0022-2860(98)00650-4

Vogler M, Bender M, Plachetka U et al (2007) Low viscosity and fast curing polymer system for UV-based nanoimprint lithography and its processing. Proc SPIE. doi:10.1117/12.708507

Wang X, Albrecht A, Mai HH et al (2013) High resolution 3D NanoImprint technology: template fabrication, application in Fabry-Pérot-filter-array-based optical nanospectrometers. Microelectron Eng 110:44-51. doi:10.1016/j.mee.2013.04.038

Wolffenbuttel RF (2005) MEMS-based optical mini- and microspectrometers for the visible and infrared spectral range. J Micromech Microengineering 15:S145-S152. doi:10.1088/0960-1317/15/7/021

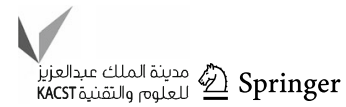

